\title{
Morfologia de sementes e de estádios iniciais de plântulas de espécies de Bromeliaceae da Amazônia ${ }^{1}$
}

\author{
Seed morphology and early seedling stages in Bromeliaceae from the Amazon
}

Ivone Vieira Silva ${ }^{2,4}$ \& Vera Lúcia Scatena ${ }^{3}$

\begin{abstract}
Resumo
Sementes de Aechmea bromeliifolia, A. castelnavii (Bromelioideae); Dyckia duckei, D. racemosa (Pitcairnioideae) e Tillandsia adpressiflora (Tillandsioideae) foram coletadas em regiões amazônicas (Mato Grosso) e estudadas visando sua caracterização morfológica e o desenvolvimento pós-seminal com finalidades taxonômicas, além de verificar a porcentagem degerminação. Todas as espécies apresentam germinação epígea eplântulas criptocotiledonares. As sementesnão apresentam dormência e a porcentagem de germinação é alta, acima de $86 \%$, facilitando a produção de mudas e estudos de conservação. Como característica exclusiva dos gêneros, o envoltório das sementes de Aechmea (Bromelioideae) apresenta mucilagem que evita a dessecação; enquanto que o de Dyckia (Pitcairnioideae) apresenta alas membranáceas e o de Tillandsia (Tillandsioideae) apresenta apêndices plumosos, neste último caso provavelmente para facilitar a dispersão e estabelecer o hábito epifítico. O início do desenvolvimento pós-seminal de Aechmea (Bromelioideae) e Dyckia (Pitcairnioideae) é marcado pela emergência da raiz primária, interpretado como caráter basal, enquanto que o de Tillandsia adpressiflora (Tillandsioideae) é marcado pela emergência do cotilédone, interpretado como caráter derivado. Dyckia e Tillandsia apresentam pequeno tanque apenas na fase de plântula e em Aechmea ocorre o contrário.
\end{abstract}

Palavras-chave: Aechmea, Dyckia, germinação, semente, Tillandsia.

\begin{abstract}
Seeds of Aechmea bromeliifolia, A. castelnavii (Bromelioideae); Dyckia duckei, D. racemosa (Pitcairnioideae) and Tillandsia adpressiflora (Tillandsioideae) were collected in the Amazon regions (Mato Grosso) and studied to describe morphological characterization and post-seminal development, which can be taxonomically useful, and to assess percent germination. All the species have epigeous germination and produce cryptocotyledonary plantlets. Seeds have no dormancy and percent germination is high (over $86 \%$ ), which facilitates the production of seedlings and conservation studies. Exclusive characteristics of the genera include: the seed coat of Aechmea (Bromelioideae) has mucilage that prevents desiccation; whereas that of Dyckia (Pitcairnioideae) has membranaceous wings and that of Tillandsia (Tillandsioideae) has feathery appendages, both of which make dispersal easier and establish the epiphytic habit. Initial post-seminal development of Aechmea (Bromelioideae) and Dyckia (Pitcairnioideae) is marked by the emergence of primary roots, interpreted as a basal character, whereas that of Tillandsia adpressiflora (Tillandsioideae) is marked by the emergence of the cotyledon, interpreted as a derived character. Dyckia and Tillandsia have a small tank only in the seedling phase while the contrary occurs in Aechmea.
\end{abstract}

Key words: Aechmea, Dyckia, germination, seed, Tillandsia.

\section{Introdução}

Bromeliaceae é uma das 16 famílias pertencentes à ordem Poales (APG III 2009) e apresenta cerca de 3.086 espécies distribuídas em 56 gêneros (Luther 2006). Suas espécies ocorrem nas mais variadas condições de altitude, temperatura e disponibilidade hídrica (Dahlgren et al. 1985). Com exceção de Pitcairnia feliciana (A. Chev.) Harms \& Mildbr. que ocorre no oeste do continente africano, as demais espécies da família são essencialmente neotropicais (Smith \& Downs 1974; Cronquist 1981).

\footnotetext{
Parte da tese de Doutorado da primeira autora.

${ }^{2}$ Universidade do Estado de Mato Grosso - UNEMAT, Depto. Biologia, Av. Perimetral Rogério Silva, C.P. 324, 78580-000, Alta Floresta, MT, Brasil.

${ }^{3}$ Universidade Estadual Paulista - INESP, Instituto de Biociências, Depto. Botânica, Av. 24-A, 1515, Bela Vista, 13506-900, Rio Claro, SP, Brasil.

${ }^{4}$ Autor para correspondência: ivibot@hotmail.com
} 
Os representantes da família apresentam em geral inflorescências vistosas e folhas distribuídas em roseta, usualmente com bainha alargada na base, propiciando a formação de um reservatório de água e nutrientes em muitas espécies (Cronquist 1981), importante na nutrição das Bromeliaceae e como micro ambiente para animais diversos (Oliveira 2004).

A importância econômica da família está na utilização como plantas alimentícias, como produtoras de fibras e como ornamentais, sendo cultivadas e utilizadas em decoração de interiores e em projetos paisagísticos (Pereira 1988; Nara \& Weber 2002; Santos et al. 2005; Duarte et al .2009). Segundo os autores, as Bromeliaceae da Amazônia estão ameaçadas de extinção pelo intenso desmatamento, que pode gerar perda da diversidade e do banco genético.

Embora a semente não seja o principal meio de propagação das espécies herbáceas de monocotiledôneas, ela é importante evento biológico (Tillich 1995). A partir do conhecimento de sua estrutura, é possível obter informações que auxiliem em estudos de germinação, armazenamento e métodos de cultivo (Pereira 1988; Andrade et al. 2003), além de auxiliar estudos sobre regeneração de ecossistemas naturais (Melo \& Varela 2006).

Para espécies de monocotiledôneas, as características de plântulas foram utilizadas como ferramenta na sistemática do grupo (Tillich 1995, 2000, 2007). De um modo geral, os tipos de plântulas representam uma adaptação funcional ao ambiente e estão associados a fatores ecológicos como: forma de vida da planta, capacidade de dispersão das sementes e estratégias de regeneração (Garwood 1996; Ibarra-Manríquez et al. 2001).

Estudos sobre morfologia de sementes e plântulas com vários representantes de Bromeliaceae forneceram informações importantes para circunscrição infragenérica e infrafamiliar (Pereira 1988; Tillich 1995, 2000, 2007; Strehl \& Beheregaray 2006; Scatena et al. 2006; Pereira et al. 2008).

Nesse contexto, foram escolhidas para estudo as espécies Aechmea bromeliifolia (Rudge) Baker, Aechmea castelnavii Baker (Bromelioideae), Dyckia duckei L.B. Smith, Dyckia racemosa Baker (Pitcairnioideae) e Tillandsia adpressiflora Mez. (Tillandsioideae) que ainda não foram estudadas e são abundantes no Parque Estadual Cristalino, localizado na Amazônia Meridional, nos municípios de Alta Floresta - MT e Novo Mundo - MT. O objetivo do trabalho foi estudar a germinação, morfologia das sementes e do desenvolvimento pós-seminal, visando levantar caracteres importantes para a taxonomia do grupo.
Os dados obtidos também poderão contribuir para futuros estudos sobre conservação das espécies.

\section{Material e Métodos}

As sementes das espécies em estudo (Aechmea bromeliifolia (Rudge) Baker, Aechmea castelnavii Baker, Dyckia duckei L.B. Smith, Dyckia racemosa Baker e Tillandsia adpressiflora Mez) foram coletadas no Parque Estadual Cristalino, municípios de Alta Floresta e Novo Mundo, ambos no estado de Mato Grosso, Brasil. As exsicatas estão depositadas no Herbário da Universidade Estadual de Mato Grosso (HERBAM).

Para a descrição biométrica das sementes (comprimento, largura e espessura) foram escolhidas, ao acaso, 50 sementes de pelo menos cinco indivíduos diferentes, medidas individualmente com paquímetro digital Mitutoyo.

Foram colocadas para germinar 200 sementes de cada espécie, distribuídas em oito repetições de 25 sementes. As sementes foram distribuídas sobre duas camadas de papel filtro em caixa gerbox e irrigadas com $1 \mathrm{ml}$ de água destilada. As caixas foram colocadas em germinadores a $25^{\circ} \mathrm{C}$, com fotoperíodo de oito horas, utilizando lâmpadas fluorescentes do tipo luz do dia $(4 \times 20 \mathrm{w})$. Antes de serem colocadas para germinar, as sementes de Aechmea Ruiz \& Pav. foram submetidas a lavagem em água corrente até a completa remoção do envoltório de mucilagem.

A emergência da raiz primária ou do cotilédone foi o critério usado para definir germinação (Pereira et al. 2008). Os cálculos de porcentagem e índice de velocidade de germinação foram realizados de acordo com Labouriau \& Valadares (1976) e Maguire (1962), respectivamente: $\mathrm{G}(\%)=(\mathrm{N} / \mathrm{A}) \times 100$, onde $\mathrm{N}=$ Número de sementes germinadas e $\mathrm{A}=$ número total de sementes; e IVG $=\Sigma(\mathrm{Gi} / \mathrm{ni})$, onde $\mathrm{Gi}=$ número de sementes germinadas e $\mathrm{ni}=$ dia da contagem.

A observação do desenvolvimento pósseminal foi feita diariamente e as ilustrações realizadas com o auxílio de estereomicroscópio óptico, equipado com câmara clara. Critérios adotados para estádio de plântula foram: desenvolvimento radicular com expansão total da primeira folha e aparecimento da segunda folha. Para planta jovem, foi considerado a expansão total da segunda e o aparecimento da terceira folha.

Atendidos os pressupostos de normalidade e homocedasticidade, os dados de biometria e germinação (porcentagem e IVG) foram submetidos à análise de variância e as médias comparadas pelo teste de Tukey 
$(\mathrm{p}=0,05)$, sendo os dados de germinação previamente transformados em arcoseno. Todas as análises foram realizadas pelo programa estatístico Sisvar® para Windows® (Ferreira 2004).

\section{Resultados}

Aechmea castelnavii (Fig. 1a) e A. bromeliifolia (Fig. 1b) apresentam sementes elípticas, com porção micropilar mais estreita; com médias aproximadas de 3,87 mm de comprimento e 1,60 mm de largura. $\mathrm{O}$ envoltório das sementes é liso, com presença de substância mucilaginosa. Aechmea bromeliifolia e A. castelnavii apresentaram em média 99 e $96 \%$ de germinação e índice de velocidade (IVG) de 4,1 e 3,95, respectivamente (Tab. 1).

Dyckia duckey (Fig. 1c) e D. racemosa (Fig. 1d) apresentam sementes ovaladas, achatadas, discóides; com médias de aproximadamente 4,65 mm de comprimento e 6,12 mm de largura. Apresentam envoltório rugoso, com alas membranáceas e circulares em uma das extremidades. A região que delimita o embrião apresenta coloração mais escura. Dyckia duckey e D. racemosa apresentaram em média $92 \%$ e $86 \%$ de germinação e IVG de 5,2 e 4,8, respectivamente (Tab. 1).

Tillandsia adpressiflora (Fig. 1e) apresenta sementes fusiformes; com cerca de 3,60 mm de comprimento e 0,60 $\mathrm{mm}$ de largura. Apresenta envoltório rugoso, com apêndices plumosos (Fig. 1e, 4a). Esses apêndices são filiformes, esbranquiçados, ligados à porção micropilar (Fig. 1e, 4a). T. adpressiflora apresentou médias de $97 \%$ de germinação e IVG de 2,8 (Tab. 1).

Todas as espécies estudadas apresentam germinação epígea e o cotilédone não se desprende do tegumento da semente, mantendo sua função haustorial, originando plântulas criptocotiledonares (Fig. 2d-h, 3d-h, 4d-f).

A germinação inicia-se após quatro a cinco dias de embebição em Aechmea bromeliifolia e $A$. castelnavii, pelo rompimento do envoltório e emissão da raiz primária na região da micrópila (Fig. 2a). A raiz primária é esbranquiçada e cônica (Fig. $2 \mathrm{a}-\mathrm{c})$. As raízes adventícias crescem antes do aparecimento do eofilo (Fig. 2e-f). Nas duas espécies de Aechmea, o eofilo cresce entre dois a quatro dias após a germinação e apresenta-se levemente lanceolado, de ápice ligeiramente acuminado e bordo inteiro (Fig. 2g). A bainha cotiledonar é foliácea, se rompe no ápice; o colo é demarcado, com hipocótilo conspícuo e cilíndrico (Fig. 2d-f).

Com aproximadamente três a cinco dias de germinação as plântulas de Aechmea apresentam
Tabela 1 - Porcentagem e velocidade de germinação de sementes de espécies de Bromeliaceae. * IVG índice de velocidade de germinação. (Médias seguidas pelas mesmas letras maiúsculas na coluna não diferem entre si pelo teste de Tukey a $0,05 \%$ de significância)

Table 1 - Percentage and speed of germination of seeds of species of Bromeliaceae. * IVG - speed of germination. (Means followed by same letters are not different by Tukey's test, $p<0.05$ )

\begin{tabular}{lcc}
\hline Espécies & Geminação(\%) & IVG* \\
\hline Aechmea bromeliifolia & $99,0 \mathrm{~A}$ & $4,10 \mathrm{C}$ \\
Aechmea castelnavii & $96,0 \mathrm{~A}$ & $3,95 \mathrm{D}$ \\
Dyckia duckey & $92,0 \mathrm{AB}$ & $5,20 \mathrm{~A}$ \\
Dyckia racemosa & $86,0 \mathrm{~B}$ & $4,80 \mathrm{~B}$ \\
Tillandsia adpressiflora & $97,0 \mathrm{~A}$ & $2,80 \mathrm{E}$ \\
\hline
\end{tabular}

raiz principal robusta, cônica, pilosa, com início de formação de raízes adventícias (Fig. 2g, 2i). O hipocótilo é longo e a bainha cotiledonar é membranosa, cupuliforme ou arqueada, frequentemente reflexas, com escamas (Fig. 2g, 2i). As plantas jovens apresentam hipocótilo cilíndrico, raízes adventícias e folhas elevadas pelos entrenós longos (Fig. 2h-k).

Em Dyckia duckey e D. racemosa a germinação inicia-se após três a quatro dias de embebição, com o rompimento do envoltório (Fig. 3a-c) na região micropilar e emissão da raiz primária, que é cônica, com pelos absorventes (Fig. 3c-d). Após um a dois dias de germinação a bainha cotiledonar se rompe no ápice e cresce o eofilo que é largo e cupuliforme, com escamas (Fig. 3d-f). A plântula (cinco a seis dias após a germinação) forma um pequeno tanque, apresentando eofilo com bainha larga e sobreposta com a da segunda folha (Fig. 3e-g). Apresenta raiz principal em processo de necrose, densamente pilosa, com bainha cotiledonar evidente (Fig. 3e-g).

As plântulas das Dyckia estudadas com seis a oito dias após a germinação apresentam bainha cotiledonar foliácea, cupuliforme (Fig. 3h). A planta jovem, após nove dias de germinação, apresenta colo delimitado e raiz primária curta (Fig. 3h-j). O hipocótilo é reduzido, com folhas em roseta e início do crescimento de raízes adventícias (Fig. 3h-i). A bainha cotiledonar é foliácea, cupuliforme, com margem ondulada e fendida (Fig. 3e-h). As folhas subsequentes são dispostas em roseta (Fig. 3g-j), com escamas no limbo e bordos espinhosos. 

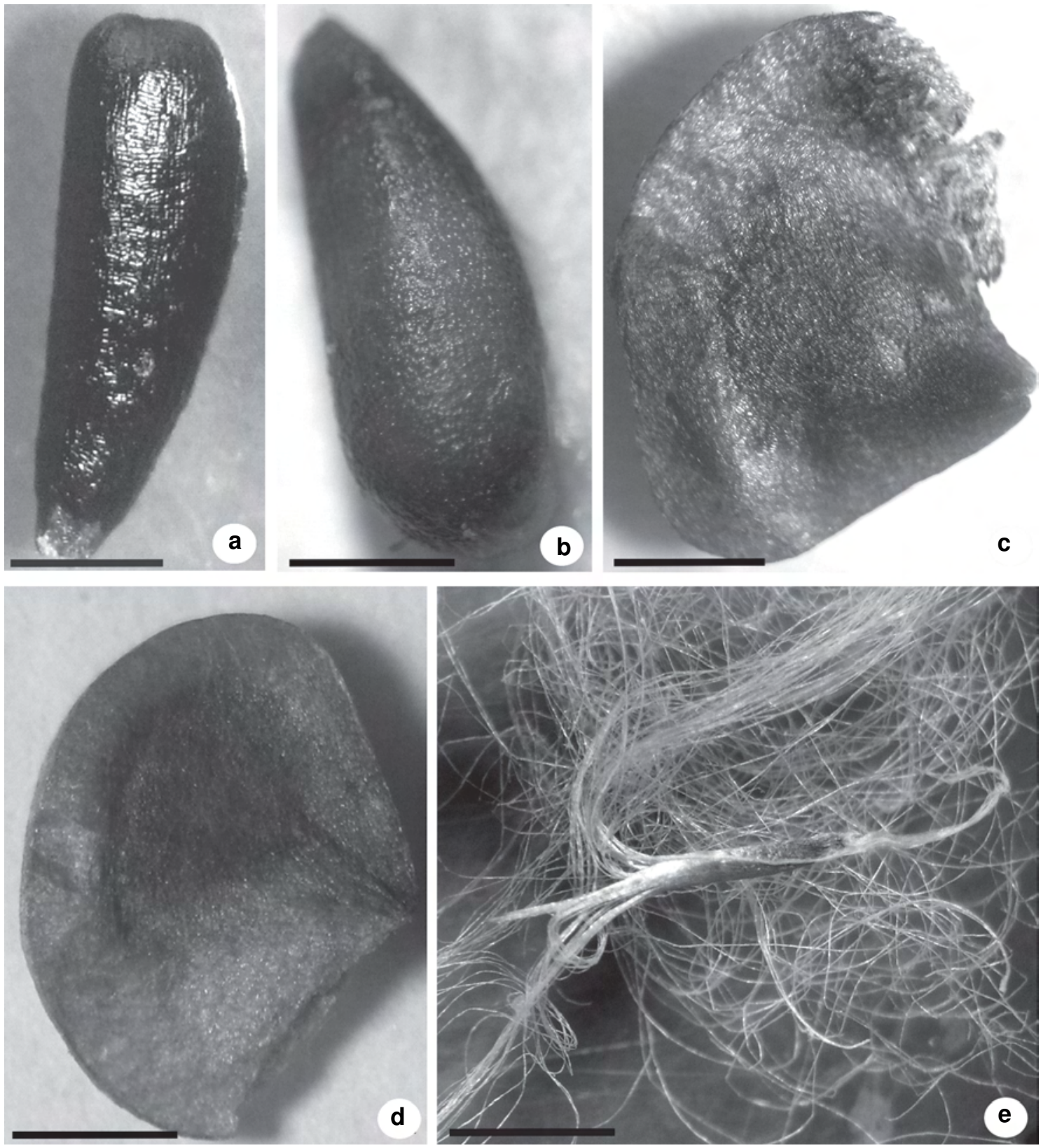

Figura 1 - Aspectos morfológicos das sementes - a. Aechmea castelnavii; b. A.bromeliifolia; c. Dyckia duckei; d. D. racemosa; e. Tillandsia adpressiflora. Barras =1,5 mm (1); $1 \mathrm{~mm}(2,3,4) ; 3 \mathrm{~mm}(5)$.

Figure 1 - Morphological aspects of seeds - a. Aechmea castelnavii; b. A. bromeliifolia; c. Dyckia duckei; d. D. racemosa; e. Tillandsia adpressiflora. Bars $=1,5 \mathrm{~mm}(1) ; 1 \mathrm{~mm}(2,3,4) ; 3 \mathrm{~mm}(5)$.

Em Tillandsia adpressiflora, após oito dias de embebição, a semente inicia a germinação, que se dá através da emergência do cotilédone haustorial na região micropilar (Fig. 4b-c) e não forma raiz primária. $\mathrm{O}$ haustório é longo e tubular (Fig. 4c). Após oito dias de germinação emerge o eofilo (Fig. 4d-e). Com nove a dez dias após a germinação o eofilo apresenta-se clorofilado, suculento, cupuliforme, com ápice acuminado, bordo inteiro, margem flexionada e bainha larga (Fig. 4e-f). Na plântula ocorre pequeno tanque, formado pela base da bainha do eofilo que fica superposta 

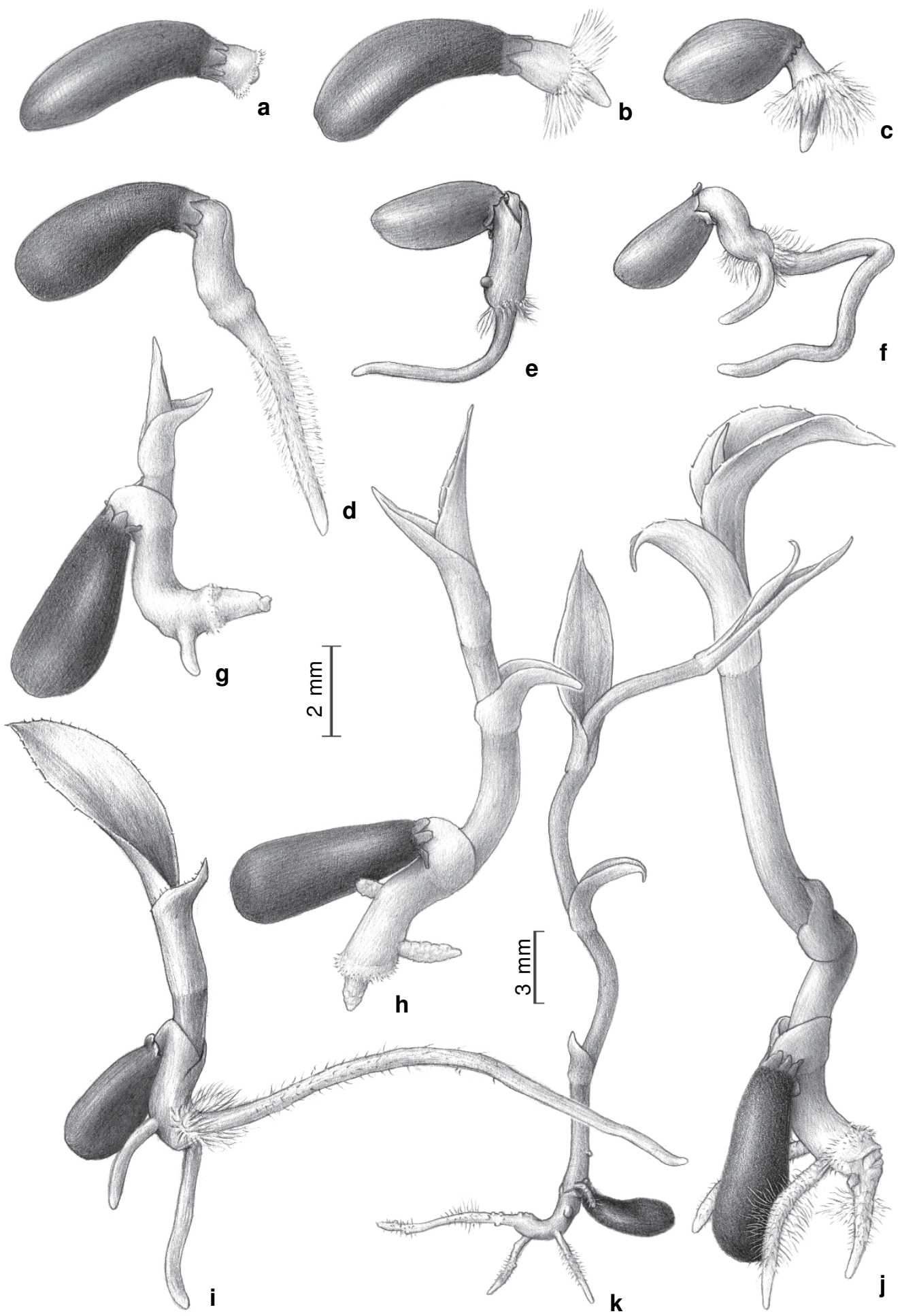

Figura 2 -Desenvolvimento pós-seminal de espécies de Aechmea (Bromelioideae, Bromeliaceae) - a-c. fases da germinação; g-i. plântulas; h, j-k. plantas jovens. a-b, d, g-h, j-k. Aechmea castelnavii. c, e-f, i. A. bromeliifolia . Barras $=2 \mathrm{~mm}(\mathrm{a}-\mathrm{j}) ; 3 \mathrm{~mm}(\mathrm{k})$. Figure 2 - Post-seminal development of species of Aechmea (Bromelioideae Bromeliaceae) - a-c. stages of germination; g-i. seedlings; h, j-k. young plant. a-b, d, g-h, j-k. Aechmea castelnavii. c, e-f, i.A. bromeliifolia. Bars $=2 \mathrm{~mm}(\mathrm{a}-\mathrm{j}) ; 3 \mathrm{~mm}(\mathrm{k})$. 


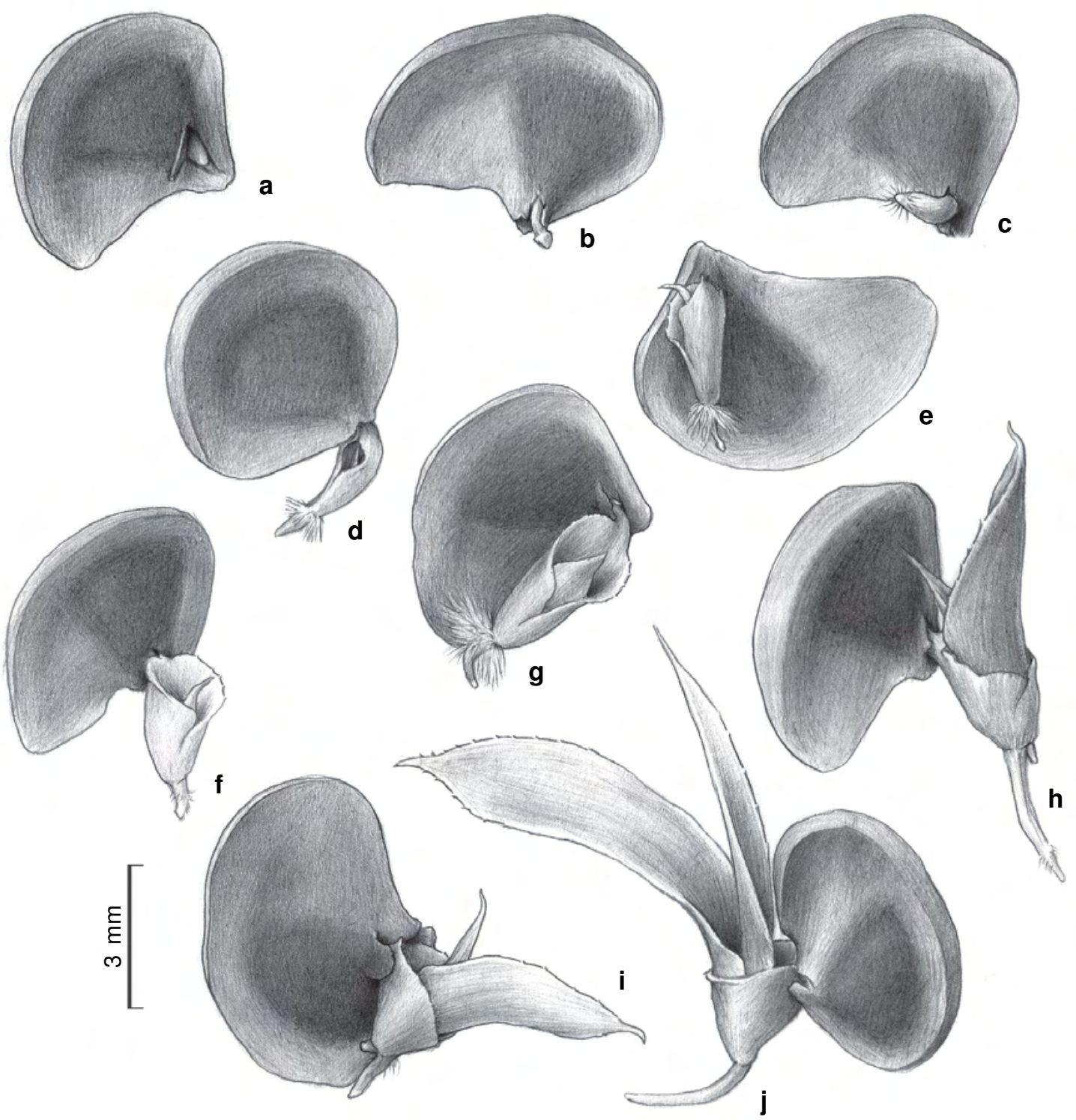

Figura 3 - Desenvolvimento pós-seminal de espécies de Dyckia (Piticairnioideae, Bromeliaceae) - a-c. fases da germinação; d-g. plântulas; h-j. plantas jovens. a, d, f-h, j. Dyckia racemosa. b-c, e, i. D. duckei. Barras = 3 mm (a-j). Figure 3 - Post-seminal development of species of Dyckia (Pticairnioideae, Bromeliaceae) - a-c. stages of germination; d-g. seedlings; h-j. young plant. a, d, f-h, j. Dyckia racemosa. b-c, e, i. D. duckei. Bars $=3 \mathrm{~mm}$ (a-j).

com a base da bainha do eofilo secundário (Fig. 4f). $\mathrm{O}$ eofilo secundário emerge após cerca de 15 dias de germinação e apresenta as mesmas características morfológicas que o eofilo primário (Fig. 4f). A planta jovem (a partir dos 32 dias após a germinação) apresenta início de formação de raízes adventícias, folhas lanceoladas, de ápices ligeiramente acuminados e filotaxia alterna, com entrenós alongados (Fig. 4g).

\section{Discussão}

As sementes das espécies aqui estudadas apresentam adaptações facilitadoras da dispersão, evidenciadas na sua morfologia: as sementes de Aechmea (Bromelioideae) apresentam mucilagem, as de Dyckia (Pitcairnioideae) apresentam ala membranácea e a de Tillandsia (Tillandsioideae) apêndices plumosos. Esses caracteres também foram 


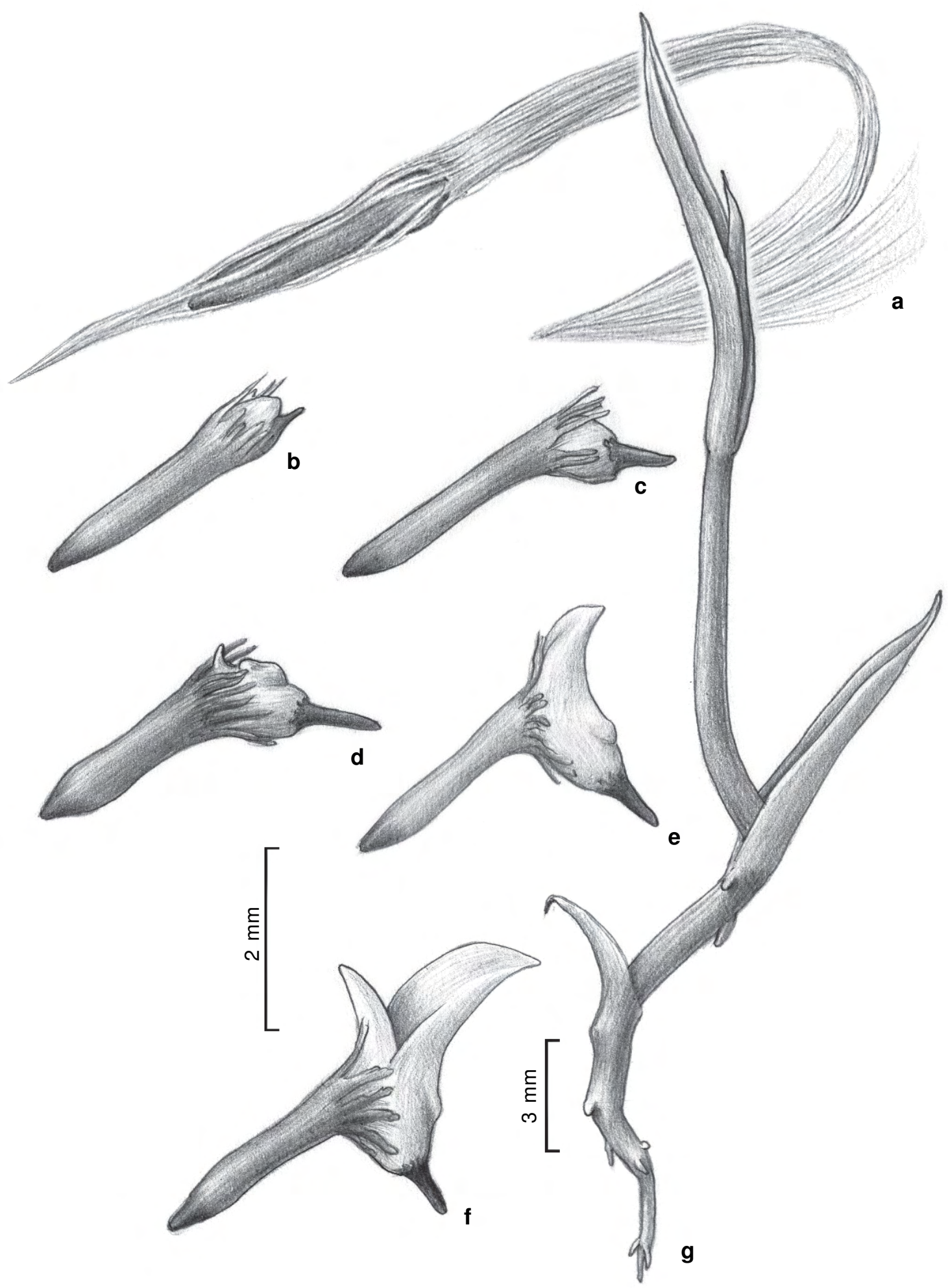

Figura 4 - Desenvolvimento pós-seminal de Tillandsia adpressiflora (Tillandsioideae, Bromeliaceae) - a. semente com apêndices plumosos; b-d. fases da germinação; e-f. plântulas; g. planta jovem. Barras =2 mm (a-f); 3 mm (g). Figure 4 - Post-seminal development of species of Tillandsia adpressiflora (Tillandsioideae, Bromeliaceae) - a. seeds with feathery appendages; b-d. stages of germination; e-f. seedlings; g. young plant. Bars = 2 mm (a-f); 3 mm (g). 
encontrados em sementes de outros representantes de Bromelioideae, Pitcairnioideae e Tillandsioideae já estudados (Pereira 1988; Varadarajan \& Gilmartin 1988; Scatena et al. 2006; Pereira et al. 2008) e confirmam-se como caracteres de importância infrafamíliar.

A mucilagem encontrada nas sementes de Aechmea (Bromelioideae) provavelmente protegem contra a dessecação, enquanto que as de Dyckia (Pitcairnioideae) e a de Tillandsia (Tillandsioideae) que apresentam sementes menores, mais leves, provavelmente são transportadas pelas correntes de ar nos períodos secos, facilitando sua dispersão. Apêndices plumosos como ocorrem em Tillandsia, auxiliam a fixação em troncos e cascas de árvores, garantindo o sucesso de sua dispersão em hábito epifítico (Van der Pijl 1982; Benzing 2000; Scatena et al. 2006).

Aechmea bromeliifolia, A. castelnavii, Dyckia duckey e Tillandsia adpresssiflora apresentam alta porcentagem de germinação, com valores médios variando entre $92 \%$ e $99 \%$. Resultados semelhantes foram encontrados em outras espécies de bromélias como em Aechmea distichantha Lem. (Mercier \& Guerreiro Filho 1990), em Aechmea beeriana L.B. Sm. \& M.A. Spencer (Nara \& Webber 2002), em quatro espécies rupícolas de Bromeliaceae (Pereira et al.2009) e em Ananas ananossoides (Baker) L.B. Sm. (Anastácio \& Santana 2010). Dyckia racemosa apresentou a menor porcentagem média de germinação (86\%) das sementes entre as espécies estudadas, que apesar de menor, indica alta qualidade fisiológica e, consequentemente, alto potencial para produção de mudas (Pereira et al. 2008). A produção de mudas via sementes mantém a variabilidade genética, importante fator para futuros estudos de recuperação de áreas degradadas e reintrodução de espécies ameaçadas de extinção. A alta qualidade fisiológica de sementes também foi observada em Dyckia goehringii E. Gross \& Rauh, principalmente nas de maior tamanho, que apresentam maior germinação e vigor, originando plântulas mais vigorosas do que as sementes pequenas (Duarte et al. 2010). A germinação epígea das espécies também foi observada em outros representantes de Bromeliaceae (Pereira 1988; Pereira et al. 2008). Segundo Labouriau (1983), sob o ponto de vista botânico, consideram-se germinadas as sementes em que uma das partes do embrião emerge dos envoltórios seminais. O início da germinação nas Aechmea e Dyckia estudadas é marcado pela emergência da raiz primária, corroborando estudos de desenvolvimento pós-seminal de outras Bromelioideae e Pitcairnioideae (Pereira 1988; Pereira et al. 2008, 2009; Duarte et al. 2009, 2010).

Em Tillandsia adpressiflora o início da germinação é marcada pela emergência do cotilédone, mesmo padrão de desenvolvimento pós seminal verificado em outras espécies do gênero Tillandsia (Scatena et al. 2006) e também em Vriesea, Alcantarea e Pitcairnia (Pereira et al. 2008, 2009). Essa característica (morfologia da germinação) poderá ser útil para futuras análises cladísticas da família. Recentes análises filogenéticas baseadas em dados moleculares apontam para necessidade de extensa revisão taxonômica em Bromeliaceae (Givnish et al. 2007; Sass \& Specht 2010).

Tillich (1995) sugere que a presença de raiz primária em monocotiledôneas é provavelmente uma condição ancestral para o grupo. Nas Poaceae, a raiz primária cessa seu crescimento pouco depois da germinação e permanece na plântula apenas como resquício, indicado pelo pólo radicular (Nakamura \& Scatena 2009). A ausência completa de raiz primária em plântulas seria um dos últimos passos evolutivos em monocotiledôneas e, para Poales, sua presença foi observada na maioria das famílias (Tillich 2000, 2007). Entretanto, em Bromeliaceae, família considerada basal dentro de Poales (APG III 2009), foi observada sua presença em representantes de Bromelioideae (Pereira 1988; Pereira et al. 2008) e sua ausência em representantes de Tillandsioideae (Tillich 1995; Scatena et al. 2006).

Nas espécies de Dyckia e Aechmea estudadas a raiz primária da plântula é coberta por pelos absorventes. Para plântulas de Bromelioideae as raízes são responsáveis pela absorção de água e nutrientes (Benzing 2000). Os pelos absorventes são estruturas auxiliares na sobrevivência das Pitcairnioideae e Bromelioideae, espécies terrestres ou rupícolas, a fim de garantir seu desenvolvimento (Smith \& Downs 1974).

As escamas foliares encontradas nos estádios iniciais de plântulas talvez representem um mecanismo importante para a absorção de água e nutrientes desde o início do desenvolvimento, assim como acontece com indivíduos adultos de Bromeliaceae, principalmente das sub-famílias Bromelioideae e Tillandsioideae (Benzing et al. 1976; Pierce et al. 2001).

As plântulas das espécies de Dyckia e Tillandsia acumulam um pouco de água entre suas folhas, no início do desenvolvimento, o que não é observado em campo nas plantas adultas. O contrário ocorre com as plântulas de Aechmea. A heterofilia em Bromeliaceae foi avaliada por Adams 
\& Martin (1986), procurando entender os processos ecológicos e evolutivos em Tillandsioideae.

Pode-se considerar aqui alguns caracteres relevantes para subsidiar análises ecológicas, além de delimitar gêneros e contribuir para reavaliação taxonômica em nível infrafamiliar: a) morfologia das sementes, que em Aechmea (Bromelioideae) são elípticas e apresentam envoltório mucilaginoso; enquanto em Dyckia (Pitcairnioideae) são achatadas e o envoltório apresenta alas membranáceas; e em Tillandsia (Tillandsioideae) que são fusiformes e o envoltório com apêndices plumosos; b) morfologia das fases iniciais de desenvolvimento das plântulas, que em Dyckia e Tillandsia apresenta-se diferente do encontrado em Aechmea; c) a primeira estrutura que emerge na germinação, que em Aechmea e Dyckia é a raiz primária e em Tillandsia o cotilédone; d) o aparecimento do eofilo, que em Tillandsia adpressiflora é relativamente mais tardio.

\section{Agradecimento}

À Fundação de Amparo à Pesquisa do Estado de Mato Grosso (FAPEMAT) o auxílio à pesquisa (732482/2008). Ao CNPq a bolsa de produtividade em pesquisa à segunda autora (processo 302105/ 2007-7).

\section{Referências}

Adams, W.W. \& Martin, C.E. 1986. Heterophylly and its relevance to evolution within the Tillandsioideae. American Journal of Botany 9: 121-125.

Anastácio, M.R. \& Santana, D.G. 2010. Características germinativas de sementes de Ananas ananossoides (Backer) L.B.SM. (Bromeliaceae). Acta Scientiarum. Biological Sciences 32: 195-200.

Andrade, A.C.S.; Cunha, R.; Souza, A.F.; Reis, R.B. \& Almeida, K.J. 2003. Physiological and morphological aspects of seed viability of a neotropical savannah tree, Eugenia dysenterica DC. Seed Science and Technology 31: 125-137.

APG - Angiosperm Phylogeny Group. 2009. An update of the Angiosperm Phylogeny Group classification for the order and families of flowering plants: APG III. Botanical Journal of the Linnean Society 161: 105-121.

Benzing, D.H. 2000. Bromeliaceae: profile of an adaptive radiation. Cambridge University Press, Cambridge. $690 \mathrm{p}$.

Benzing, D.H.; Henderson, K.; Kessel, B. \& Sulak, J. 1976. The absorptive capacities of bromeliad trichomes. American Journal of Botany 63: 1009-1014.

Cronquist, A. 1981. An integrated system of classification of flowering plants. Columbia University Press, New York. 1262p.
Dahlgren, R.M.T.; Clifford, H.T. \& Yeo, P.F. 1985. The families of the monocotyledons. Structure, evolution, and taxonomy. Springer-Verlag, Berlin. 520p.

Duarte, E.F.; Carneiro, I.F. \& Rezende, M.H. 2009. Morfologia de frutos e sementes e desenvolvimento pós-seminal de Dyckia goehringii Gross \& Rauh (Bromeliaceae). Revista Biologia Neotropical 6: 1-12.

Duarte, E.F.; Carneiro, I.F.; Silva, N.F. \& Guimarães, N.N.R. 2010. Características físicas e germinação de sementes de Dyckia goehringii Gross \& Rauh (Bromeliaceae) sob diferentes temperaturas. Pesquisa Agropecuária Tropical 40: 422-429.

Ferreira, D.F. 2004. Sisvar-sistema de análise de variância para dados balanceados. Versão 4.6. Universidade Federal de Lavras, Lavras. 32p.

Garwood, N.C. 1996. Functional morphology of tropical tree seedlings. In: Swaine, M.D. (ed.). The ecology of tropical forest tree seedlings. Man and the Biosphere series, Paris. Pp. 59-129.

Givnish T.J.; Millam K.C.; Berry P.E. \& Sytsma K.J. 2007. Phylogeny, adaptive radiation, and historical biogeography of Bromeliaceae inferred from $n d h F$ sequence data. In: Columbus, J.T.; Friar, E.A.; Porter, J.M.; Prince, L.M. \& Simpson, M.G. (eds.). Monocots: comparative biology and evolution Poales. Rancho Santa Ana Botanic Garden, Claremont. Pp. 3-26.

Ibarra-Manríquez, G.; Ramos, M.M. \& Oyama, K. 2001. Seedling functional types in a lowland rain forest in México. American Journal of Botany 88: 1801-1812.

Labouriau, L.G. 1983. A germinação das sementes. Secretaria Geral da Organização dos Estados Americanos, Programa Regional de Desenvolvimento Científico e Tecnológico, Washington. 173p.

Labouriau, L.G. \& Valadares, M.E.B. 1976. On the germination of seeds Calotropis procera (Ait.) Ait.f. Anais da Academia Brasileira de Ciências 48: 263-284.

Luther H.E. 2006. An alphabetical list of Bromeliad binomials. The Bromeliad Society International, Sarasota. 119p.

Maguire, J.D. 1962. Speeds of germination-aid and evaluation for seedling emergence and vigor. Crop Science 2: 176-777.

Melo, M.F.F. \& Varela, V.P. 2006. Aspectos morfológicos de frutos, sementes, germinação e plântulas de duas espécies florestais da Amazônia: Dinizia excelsa Ducke (Angelim Pedra) e Cedrelinga catenaeformis Ducke (Cedrorana) Leguminosae: Mimosoideae. Revista Brasileira de Sementes 28: 54-62.

Mercier, H. \& Guerreiro Filho, O. 1990. Propagação sexuada de algumas bromélias nativas da Mata Atlântica: efeito da luz e da temperatura na germinação. Hoehnea 17: 19-26.

Nakamura, A.T. \& Scatena. V.L. 2009. Desenvolvimento pós-seminal de espécies de Poaceae (Poales). Acta Botanica Brasilica 23: 212-222. 
Nara, A.K. \& Webber, A.C. 2002. Biologia floral e polinização de Aechmea beeriana (Bromeliaceae) em vegetação de baixio na Amazônia Central. Acta Amazonica 32: 571-588.

Oliveira, R.R. 2004. Importância das bromélias epífitas na ciclagem de nutrientes da Floresta Atlântica. Acta Botanica Brasilica 18: 793-799.

Pereira, T.S. 1988. Bromelioideae (Bromeliaceae): Morfologia de desenvolvimento pós-seminal de algumas espécies. Arquivos do Jardim Botânico do Rio de Janeiro 29: 115-154.

Pereira, A.R.; Pereira, T.S.; Rodrigues, A.S. \& Andrade, A.C.S. 2008. Morfologia de sementes e do desenvolvimento pós-seminal de espécies de Bromeliaceae. Acta Botanica Brasilica 22: 1150-1162.

Pereira, A.R.; Andrade, A.C.S.; Pereira, T.S. Forzza; R.C. \& Rodrigues, A.S. 2009. Comportamento germinativo de espécies epífitas e rupícolas de Bromeliaceae do Parque Estadual do Ibitipoca, Minas Gerais, Brasil. Revista Brasileira de Botânica 32: 827-838.

Pierce, S., Maxwell, K., Grifftiths, H. \& Winter, K. 2001. Hydrophobic trichome layers and epicuticular wax powders in Bromeliaceae. American Journal of Botany 88: 1371-1389.

Santos, A.J.; Bittencourt, A.M.\& Nogueira, A.S. 2005. Aspectos econômicos da cadeia produtiva das bromélias na região metropolitana de Curitiba e litoral Paranaense. Floresta 35: 409-417.

Sass, C. \& Specht, C.D. 2010. Phylogenetic estimation of the core Bromelioids with an emphasis on the genus Aechemae (Bromeliaceae). Molecular Phylogenetics and Evolution 55: 559-571.

Scatena, V.L.; Segecin, S. \& Coan, A.I. 2006. Seed morphology and post-seminal development of Tillandsia L. (Bromeliaceae) from the "Campos Gerais", Paraná, Southern Brazil. Brazilian Archives of Biology and Technology 49: 945-951.

Smith, L.B. \& Downs, R.J. 1974. Pitcairnioideae (Bromeliaceae). Flora Neotropica, Monograph 14: 1-662.

Strehl, T. \& Beheregaray, R.C.P. 2006. Morfologia de sementes do gênero Dyckia, subfamília Pitcairnioideae (Bromeliaceae). Pesquisas Botânicas 57: 103-120.

Tillich, H.J. 1995. Seedlings and systematics in monocotyledons. In: Rudall, P.J.; Cribb, P.J.; Cutler, D.F. \& Humphries, C.J. (eds.). Monocotyledons: systematics and evolution. Kew Botanic Garden, Kew. Pp. 303-352.

Tillich, H.J. 2000. Ancestral and derived character states in seedlings of monocotyledons. In: Wilson, K.L. \& Morrison, D.A. (eds.). Monocotyledons: systematics and evolution. CSIRO, Melbourne. Pp. 221-228.

Tillich, H.J. 2007. Seedling diversity and homologies of seedling organs in the order Poales (Monocotyledons). Annals of Botany 100: 1-17.

Van Der Pilj, L. 1982. Principals of dispersal in higher plants. 3rd ed. Springer Verlag, Berlin. 214p.

Varadarajan, G.S. \& Gilmartin, A.J. 1988. Taxonomic realignments within the subfamily Pitcairnioideae (Bromeliaceae). Systematic Botany 13: 294-299. 